\title{
On the correlation between average velocities of galaxies and their average luminosities in the closest large clusters of galaxies
}

\author{
H. A. Harutyunian*1, A. M. Grigoryan ${ }^{\dagger 1}$, and A. Khasawneh ${ }^{\ddagger 2}$ \\ ${ }^{1}$ NAS RA V. Ambartsumian Byurakan Astrophysical Observatory (BAO), Armenia \\ ${ }^{2}$ Royal Jordanian Geographical Center, Jordan
}

\begin{abstract}
The evolution of baryonic matter caused by the interaction with the dark energy carrier and its possible influence on the spectral characteristics of atoms are considered. Taking into account the earlier conclusion about the secular growth of mass of atomic nuclei and (perhaps) elementary particles due to interaction with the dark energy carrier, we reach a conclusion that in the course of evolution the spectral series of chemical elements should undergo blueshift. The longer is the evolution path, the higher is blueshift. We show here that galaxies of the two nearest clusters of galaxies exhibit a luminosity-redshift negative correlation which was expected in the frame of this deductive reasoning.
\end{abstract}

Keywords: Galaxies: lumnosity, redshift, galaxies: giant, dwarf, metallicity.

\section{Introduction}

It is evident that all the cosmic objects and their systems evolve in the course of time. They start their lives at any moment of time, transforming from a principally different state of a proto-object, continue their lives in the new stage and then finish it completing thus their life cycles. That is the natural evolution process. Scientists may argue about possible evolutionary scenarios, about the physical essence of a proto-object, or about what it turns into, but the very existence of the life cycle of any object can hardly be in doubt.

However, the axioms of modern physics and corresponding experimental data consider representatives of a distinct type to be unchangeable in the sense of evolution. Those are the elementary particles, atomic nuclei, and atoms themselves. There is no hint explicitly evidencing the change of these objects.In fact, even if these objects actually are subjects to secular change or in some way evolve, the corresponding changes are very difficult to detect. One should find some non-trivial way of considering the problem.

What kind of evolution can undergo quantum-mechanical objects, such as elementary particles or atomic nuclei? For modern physics, all the similar particles or nuclei are indistinguishable, i.e. if one replaces one proton with another no one will notice this replacement in any way. This is one of the fundamental axioms of quantum mechanics. On the other hand, it is well known that baryons change their mass being part of the atomic nucleus to make it stable enough for existing. Moreover, the same neutron or proton losses different amounts of mass being part of different nuclei. What does it mean? First, the baryon mass variation depending on the nucleus type might mean that any baryon is changeable and changes at least its mass obeying the surrounding physical conditions. Thus, one of the most essential issues necessary to clarify for answering the question on the evolution of quantum-mechanical objects is associated with the change of physical conditions in the course of time.

\footnotetext{
*hhayk@bao.sci.am, Corresponding author

${ }^{\dagger}$ anigrigg@gmail.com

‡awni@yahoo.com
} 


\section{Evolution of baryonic matter}

In a series of papers Harutyunian (2014, 2017), Harutyunian \& Grigoryan (2018), we consistently develop the idea of the unavoidability of the physical interaction between baryonic matter and the carrier of dark energy, settling within the framework of generally accepted laws of physics. This process should have several very significant physical consequences one has to take into account when considering the associated processes. In Harutyunian \& Grigoryan (2018), we used the same ideology to interpret the growth of the Astronomical Unit (AU) hypothesizing that the dark energy carrier interacts with the baryonic matter at all scales and that the laws of thermodynamics remain valid in the process of interaction.

The logical chain based on our knowledge of physics leads then to the conclusion that owing to the mentioned interaction, if any, gradually decreases the nuclear binding energy. Such a result follows from the second law of thermodynamics if one takes into account that nuclear binding energy is a negative one and dark energy is a positive one. This means that due to the interaction of atomic nuclei with a carrier of dark energy, the latter receives a certain portion of energy and reduces the absolute value of the deficit of its energy. It is obvious, that we arrive at the same conclusion if we consider the interaction between gravitationally bound objects and the carrier of dark energy. As a primary result, in both cases, the predicted energetic change of baryonic objects gradually diminishes their stability. On the other hand, due to the decrease of nuclear binding energy one should expect an increase in nuclear mass, which results inan increase in the mass of all baryonic objects.

In Harutyunian \& Grigoryan (2018), we attempted to calculate the Sun's mass change using the observational data concerning the value of the AU annual growth. To this end, we proceeded from the fact that instead of the speed of the Earth's removal from the Sun, which we predicted using the Hubble expansion rate for the scale under consideration, one observes a value of two orders of magnitude smaller, because of the increase in the mass of the Sun. This information is enough for calculating the necessary amount of the additional mass transformed from the dark energy storage into the ordinary matter. For the Sun's mass growth we got $\Delta M \sim 7 \times 10^{-11} M_{\odot} y r^{-1}$, which is equal to $4.5 \times 10^{15} \mathrm{~g} / \mathrm{s}$. In energy units,it amounts to $4.05 \times 10^{36} \mathrm{erg} / \mathrm{s}$. One can compare this value with the Sun luminosity to obtain it in solar luminosities $\sim 1000 L_{\odot}$.

Remaining in this framework, one can mention another observational effect of the mass growth of atomic nuclei and elementary particles. One can predict this effect based on the famous formula giving the wavelength of spectral lines for hydrogen or hydrogen-like ions:

$$
\frac{1}{\lambda_{m n}}=R Z^{2}\left(\frac{1}{m^{2}}-\frac{1}{n^{2}}\right)
$$

where $\mathrm{R}$ is the Rydberg constant:

$$
R=\frac{\mu e^{4}}{8 \varepsilon_{0}^{2} h^{3} c},
$$

and $\mu=m_{e} M_{n} /\left(m_{e}+M_{n}\right)$ is the reduced mass of electron and nucleus, $m_{e}$ and $M_{n}$ are the masses of electron and nucleus. For the hydrogen atom $R=1.09677583 \times 10^{7} \mathrm{~m}^{-1}$. It is obvious that if increases either electron's, or nucleus' mass, or both of them, the Rydberg constant gets larger. It does mean, that owing to the gradual increase of the masses of elementary particles spectral lines should undergo blueshift.

Of course, the idea concerning the evolutionary blueshift is not new, it was developed more consistently in quasi-stationary cosmology. However, in the current paradigm, we can point out the mechanism of the mass growth, which we assume to be linked with the gradual mass birth due to the interaction between the ordinary baryonic matter and the carrier of dark energy. It seems that during the evolution of the baryonic Universe constantly works a self-consistent mechanism, transferring dark energy into the baryonic mass.

\section{The cosmic objects' evolution rate depending on their mass}

For evolutionary purposes, it is very important to know the consequences of the interaction between the carrier of dark energy and much larger cosmic objects. Physical intuition suggests that more 
massive objects are more difficult to be affected by dark energy than low-mass ones.Using the example of a spherical object,it is easy to verify this assumption. Such an object's gravitational potential energy is given by the following formula:

$$
U=-G \frac{M^{2}}{R} .
$$

It is evident, that the same physical justification used above for the gradual decreasing of the nuclear binding energy might be used here for the gravitational potential energy issue. Then one arrives at the conclusion that the absolute value of goes down in the course of time. Hence, one can see that in (3) either the numerator should reduce, or the denominator should enlarge.

We saw in the previous section that the mass increases due to a decrease in the binding energy in the microworld. Therefore, one can conclude, that the size of the object gets larger faster than the square of its mass. If we consider the Universe as such an object, we can conclude that its size and mass are growing. The first conclusion is obvious since the Universe is expanding. The second one follows from our deduction on the mass growth in general. There is also another cosmological interpretation for this: the much younger Universe could not have been expanding if it would have the same mass as today.

For checking the influence of dark energy on the baryonic objects of different masses one can use the ratio of the gravitational and dark energy densities. Denoting dark energy density by $\rho_{D E}$ and the mass density of the object by $\rho_{M}$, one can find that within the radius these energies ratio is

$$
k_{E}=\frac{U}{\frac{4 \pi}{3} R^{3} \rho_{D E}} \approx \frac{M}{R} \frac{\rho_{M}}{\rho_{D E}} .
$$

It is obvious from (4) that the more massive are objects, the bigger is the ratio if only the baryonic objects' density does not decrease inversely proportional to the square of the radius or faster. The objects belonging to the same type (stars or galaxies or planets), such dependence is not observed. What does it mean? This dependence can be interpreted in favor of the fact that massive objects evolve more slowly, that is, the more accumulated baryon mass, the more difficult it is for dark energy to carry it along the path of evolution.

Therefore, one can assume that objects of different masses formed together apparently would be at different stages of evolution. It does mean that for testifying the consistency of the conclusions made above, one should find the appropriate objects.

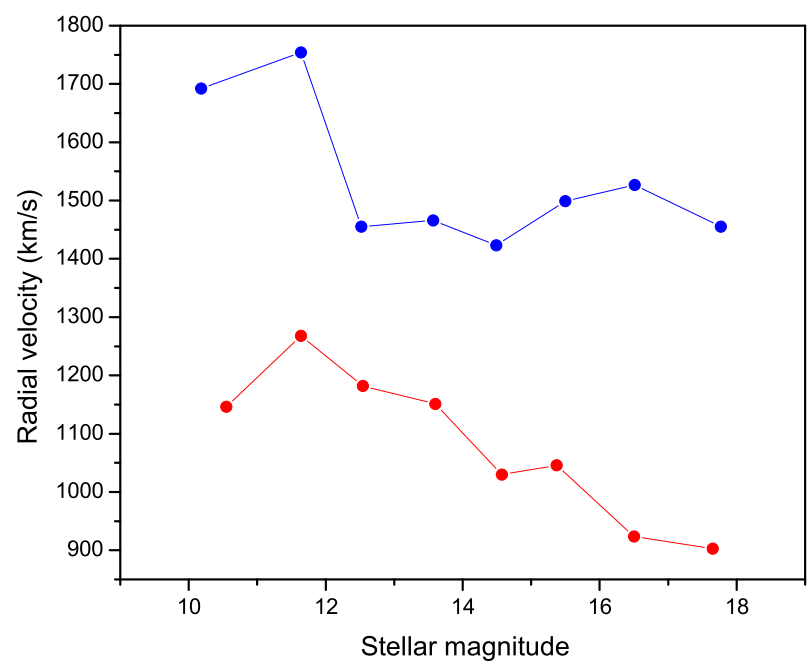

Figure 1: Radial velocities vs. stellar magnitudes for Virgo (red line) and Fornax clusters (blue line). The correlation coefficient for the 400 Virgo galaxies is -0.90 . For the Fornax 125 galaxies, the correlation coefficient is -0.64 . 


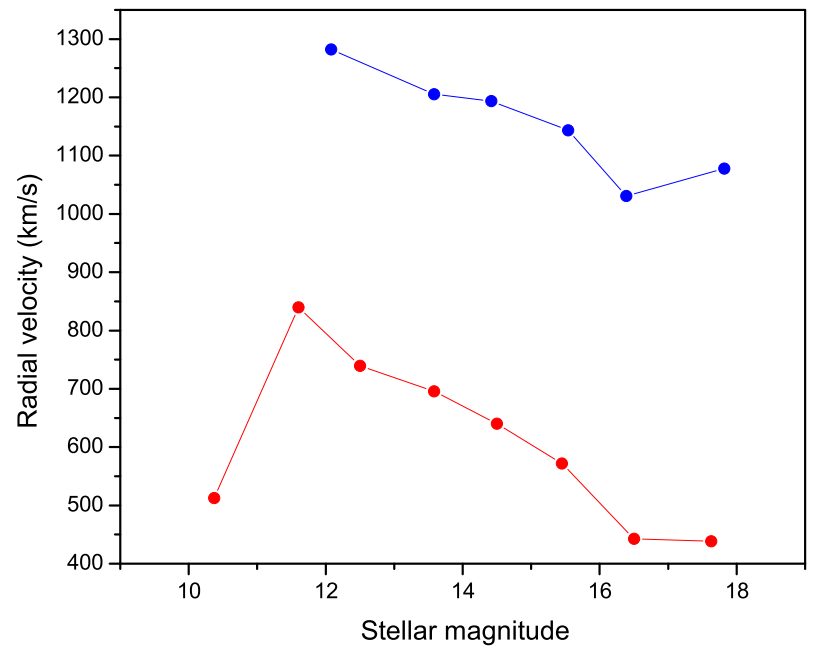

Figure 2: Radial velocities vs. stellar magnitudes for galaxies from Virgo (red line) and Fornax (blue line) clusters with radial velocities lower than the average. The correlation coefficient for the Virgo galaxies is -0.60 and for Fornax galaxies is -0.91 .

\section{Galaxy clusters}

Galaxy clusters are the best laboratories to test the conclusions derived in the previous sections of this paper. The point is that the masses of dwarf and giant galaxies can differ up to a million times. Therefore, using galaxies of various luminosities belonging to the same cluster, we can compare their baryonic matter properties, which we believe are depending on the length of the evolutionary path.

One such difference between the features of the baryonic matter of dwarf and giant galaxies is well known. We are talking about the dependence of luminosity-metallicity or, which is the same, mass-metallicity.A correlation between the galaxies metallicity and blue luminosity was demonstrated by Garnett \& Shields (1987) and extended by various authors (Brodie \& Huchra, 1991, Skillman, 1989, Zaritsky et al., 1994) to include a range of Hubble types and to span over 11 magnitudes in luminosity and 2 dex in metallicity.

Traditional cosmology explains this dependence as the selective loss of metals from galaxies with shallow potential wells via galactic winds, an idea rst introduced by Larson (1974). Nevertheless, this mechanism is not satisfactory for explaining the observed correlation. Unfortunately, one needs to construct far too artificial models for reaching the observed metallicities for the given baryonic masses.

When interpreting the mass-metallicity correlation in the frame of the paradigm we develop here,one has a much more unambiguous physical mechanism leading to the formation of different chemical abundances in the galaxies of different masses. The longer the evolution, the lower metallicity in the baryonic matter.

Indeed, taking into account the conclusion about the evolution rate dependence on the mass of a galaxy one arrives at the conclusion that the baryonic matter in dwarf galaxies easier enriches with light elements including the hydrogen. It decreases the metallicity of dwarf galaxies much rapidly than in the giant ones. So, the most essential result one derives from this logical chain is that the rate of evolution of dwarf galaxies is higher, and therefore they undergo longer evolution in course of the same time than giant ones. The longer is the evolution, the less is the metallicity.

On the other hand, as was mentioned in the previous paragraph the mass increase of atomic nuclei leads to the blueshift of the spectral series of the atoms. It does mean, that the longer is the evolution process, the shorter should be spectral lines wavelengths. If this logical chain is correct, one should expect that the redshifts of dwarf galaxies are smaller than those of giants. We checked it for two clusters, using the data published for the famous galaxy clusters in Virgo (Binggeli et al., 1985) 


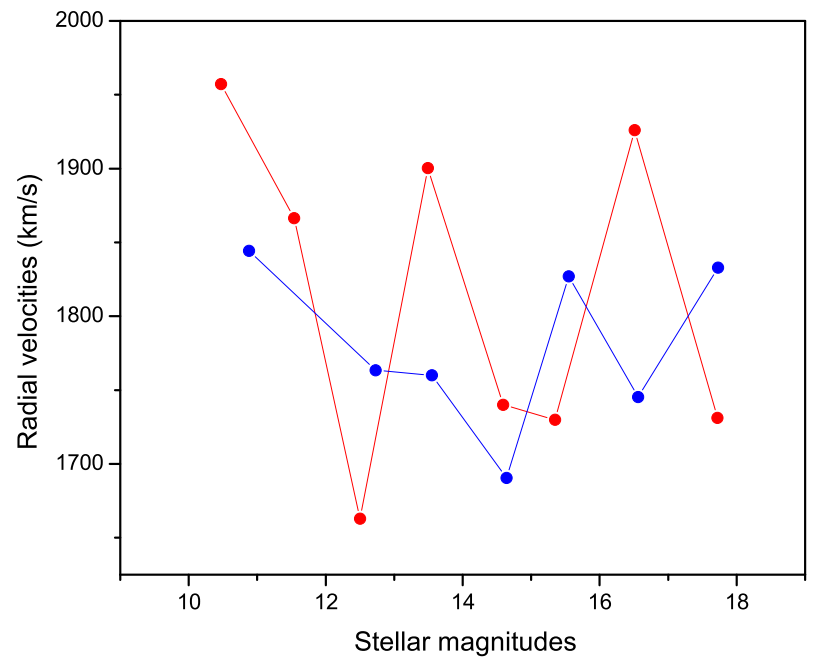

Figure 3: Radial velocities vs. stellar magnitudes for galaxies from Virgo (red line) and Fornax (blue line) clusters with radial velocities higher than the average. The correlation coefficient for the Virgo galaxies is -0.04 and for Fornax galaxies is -0.06 .

and Fornax (Ferguson, 1989). Only the galaxies marked as cluster members have been used for the analysis. All the data is taken from the mentioned papers, and no changes or corrections had been done for the data improving purposes.

In order to verify the result obtained above concerning the possible blueshift of spectral lines caused by the baryonic matter evolution, we first constructed the luminosity distribution of galaxies for both lists separately. Then the galaxies have been divided into bins of one stellar magnitude width. For each bin, the average redshift and average magnitude of galaxies were calculated. In Fig. 1 the results of these calculations are shown. At first glance, it can be seen that there is a tight negative correlation between luminosities and redshifts. The correlation coefficient for the 400 Virgo galaxies amounts -0.90 , which is really high. If one excludes the first point, which refers to the most massive galaxies, one finds for the correlation coefficient the value of -0.98. For the Fornax 125 galaxies, the correlation coefficient is -0.64 , which is also a rather high bat not as significant as for the Virgo cluster.

Next, we divided the samples of galaxies into two subsamples - having a speed lower and higher than the average of the cluster. It is interesting that the two subsamples have different behavior. The graphs are shown in Fig. 2 and Fig. 3. In both clusters, the correlation we are looking for is sharper for the galaxies possessing speeds lower than the average for the given cluster. The Virgo sample gives for the correlation coefficient $-0.60(-0.99)$ for the first subsample and -0.04 for the second subsample. The same coefficients for the Fornax subsamples are -0.91 and -0.06 , correspondingly.

One can see that the galaxies of our neighboring two large clusters show the expected luminosityredshift correlation. This correlation is caused mainly by the galaxies possessing redshifts which are lower than the cluster average redshift. In other words, the correlation is well noticeable for galaxies "blueshifted" with regard to the cluster mean recession speed.

\section{Conclusion}

Owing to the interaction with dark energy carrier the baryonic matter undergoes evolution process, changing some structural parameters, which can be manifested through observational shreds of evidence. We consider here the possible physical changes of atomic nuclei and predict blueshift of spectral series due to the evolutionary changes. Actually, one of the predicted changes is the wellknown mass-metallicity relation observed for galaxies, which finds a completely new interpretation. In order to verify or reject the second one, we were looking for a correlation between galaxy luminosities and redshifts. 
Analyzes of the relevant data for the Virgo and Fornax clusters alow to exhibit the expected correlation. It is interesting that galaxies of the chosen samples fit the predicted correlation at the expense of the subsample having lower speeds than the average for the cluster redshift. In both cases, galaxies having larger the average redshift do not show any correlation. We believe that this issue has some physical meaning which will be considered later.

\section{References}

Binggeli B., Sandage A., Tammann G. A., 1985, AJ, 90, 1681

Brodie J. P., Huchra J. P., 1991, ApJ, 379, 157

Ferguson H. C., 1989, AJ, 98, 367

Garnett D. R., Shields G. A., 1987, ApJ, 317, 82

Harutyunian H. A., 2014, in "Instability and Evolution of Stars", Proceedings of the ByurakanAbastumani Colloquium dedicated to Ludwik Mirzoyan's 90th anniversary, Eds.: Harutyunian, H. A.; Nikoghosyan, E. H.; Melikian, N. D.. pp 89-93

Harutyunian H. A., 2017, Astrophysics, 60, 572

Harutyunian H. A., Grigoryan A. M., 2018, ComBAO, 65, 268

Larson R. B., 1974, MNRAS, 169, 229

Skillman E. D., 1989, ApJ, 347, 883

Zaritsky D., Kennicutt Robert C. J., Huchra J. P., 1994, ApJ, 420, 87 